\title{
Leakage Inductance Calculation of Complex Transformer Constructions based on a Simple Two-Coil Inductor Model
}

\author{
Yick Po Chan, Student Member, IEEE \& Man Hay Pong, Senior Member, IEEE \\ The University of Hong Kong \\ Department of Electrical Engineering \\ Rm 301, Haking Wong Bldg., Pokfulam Rd, Hong Kong \\ Email: achan@eee.hku.hk
}

\begin{abstract}
The paper presents a set of systematic matrices operation derived from Kirchhoff's Node Law with the base of a simple two-coil inductor model to deduce leakage inductances of windings seen from the schematic point of view. This method provides a platform in calculating leakage inductances of complex transformer structures. Its robustness covers different kinds of concentric winding structures where by getting accurate values of mutual inductances between all combinations of winding elements in a pair-wise sense, accurate leakage inductances can be deduced through the matrices operation without any computational simulations. In essence, such operation reduces lots of difficult magnetic problems within a transformer into determination of mutual and self inductances of winding elements through a simple two-coil inductor model. The approach has been implemented on PowerESIM [5][6], a web base server type power converter design program, for reader to test.
\end{abstract}

\section{INTRODUCTION}

Nowadays transformer construction in power supply is getting more complex, especially in Switch-Mode Power Supplies where different kinds of requirements are needed to be tackled. The calculation of inductance has always been a troublesome topic when engineers deal with transformer with complex construction. Mutual inductances and more importantly, leakage inductances in an interleaved multi-winding transformer are often difficult to model as there are many limitations in existing methods that hamper the flexibility of calculations to provide accurate results. In [1] the authors proposed a lump element equivalent circuit to model multi-winding transformers. The results showed good match with actual values. However the equivalent circuit complexity is simply getting enormous as the number of windings increases, where many measurements are required and parasitic components of equivalent circuit are needed to be deduced. Refer to [2], Heinemann proposed another way of analyzing a multi-winding transformer model with an impedance network. Similar situation arises where the network builds up rapidly that this is unfavorable to any kinds of simulation software.

In power supplies industries, long design period in optimizing a transformer usually happens, as the leakage inductance is often mistreat by engineers. However, this is usually one of the most important factors in circuit performance, such as

conversion efficiency and electromagnetic interference (EMI). In order to understand better the characteristics of transformer with different kinds of winding construction, a simple robust method in a systematic way with accurate estimation of leakage inductance is essential. Here we propose a method with the general characteristic of a transformer fully determined by a closed-form matrix without any simulations. Experimental results are provided to justify the accuracy of such matrices operations.

\section{FUNDAMENTAL TRANSFORMER MODEL IN FREQUENCY DOMAIN}

\section{A. Definition of a Winding Element in a transformer}

A primary or secondary winding shown in the schematic level may consist of several winding portions in a physical transformer. An example is shown in Fig. 1 to demonstrate one practical case. Here we have a four-layer multi-winded transformer. Winding WA consists of two winding elements $\left(W_{1} \& W_{3}\right)$ connected in parallel, winding $W C$ consists of two winding elements $\left(W_{2} \& W_{4}\right)$ connected in series and winding $W B$ consists of $W_{5}$ only. A winding element $\left(W_{X}\right)$ is defined here as a continuous winding portion on a layer in a transformer. Winding element is employed as the base of a two-coil concentric inductor model described below.

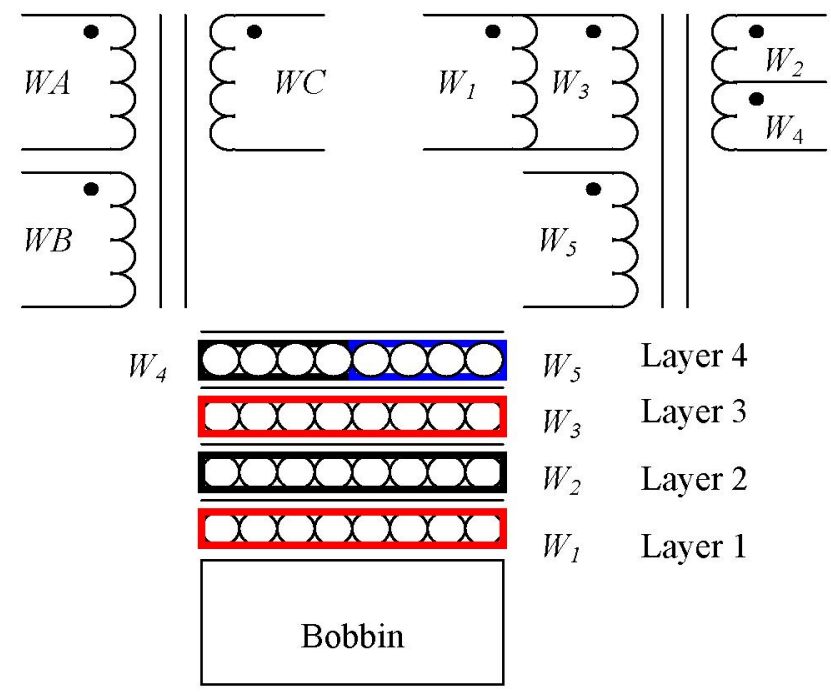

Fig. 1: A Four-Layer Multi-Winded Transformer with its schematic diagram 


\section{B. Two-coil Concentric Inductor Model}

In a complex transformer construction with many interleaved windings in a concentric construction, we can always find a mutual inductance between any pair of windings. For example, there is a mutual inductance between $W_{1} \& W_{2}$, we denote this as $M_{12}$. Also note that $M_{12}$ is the same as $M_{21}$. Thus in our example we can calculate 10 mutual inductances (i.e. $5 \mathbf{C}_{2}, 5$ winding elements $=10$ combinations) and deduce in total 20 values of mutual inductances $M_{x y}(x=1 \ldots 5, y=1$ $\ldots 5, x \neq y$ ). Moreover we can easily find the self inductances of winding elements $W_{1}, W_{2}, W_{3}, W_{4}$ and $W_{5}$ from standard formula, we denote this as $L_{z z}(z=1 \ldots 5)$.

It is known that the mutual inductance of two solenoidal windings winded concentrically essentially comes from the sum of a four-integrals formula, two positive and two negative, which are functions of the four distances measured between the ends of one coil and the ends of the other. As this absolute formula involves elliptic integrals of all three kinds, which is tedious and complicated to find, there exists a simplified way from which a look up table is used instead provided by Grover [3]. At high switching frequencies, the mutual inductance between two winding elements requires a modification factor as the so-called Skin Effect and Proximity Effect take over the uniformity of the current density in a wire, changing the effective inductance of the windings. High frequency effects on self and mutual inductance will not be considered in this paper for simplicity. Values $M_{x y}$ and $L_{z z}$ are of great importance as in later sections, it shows that these values formed the core matrix for the whole operation in order to deduce the leakage inductances of a complicated winding structure.

\section{Winding Element Matrix Model}

In our example shown in Fig. 1 , when a voltage $\boldsymbol{v}_{x}$ is applied to a winding element in the transformer, there is a current $\boldsymbol{i}_{x}$ associated with it, where the relationship in frequency domain after Laplace Transform (where $s=j \omega$ ) is given by

$$
v_{x}=s L_{x x} \cdot i_{x}+s \cdot\left[\sum_{\substack{y=1 \\ y \neq x}}^{n}\left(M_{x y} \cdot i_{y}\right)\right]
$$

Where $n$ is the number of winding elements

Thus we have a matrix to describe all voltage and current relationship for all winding elements in the form

$$
\left(\begin{array}{l}
v_{1} \\
v_{2} \\
v_{3} \\
v_{4} \\
v_{5}
\end{array}\right)=s\left(\begin{array}{lllll}
L_{11} & M_{12} & M_{13} & M_{14} & M_{15} \\
M_{21} & L_{22} & M_{23} & M_{24} & M_{25} \\
M_{31} & M_{32} & L_{33} & M_{34} & M_{35} \\
M_{41} & M_{42} & M_{43} & L_{44} & M_{45} \\
M_{51} & M_{52} & M_{53} & M_{54} & L_{55}
\end{array}\right) \cdot\left(\begin{array}{c}
i_{1} \\
i_{2} \\
i_{3} \\
i_{4} \\
i_{5}
\end{array}\right)
$$

\section{Series and Parallel Reduction Techniques}

Series and Parallel Reduction Techniques originate from Kirchhoff's Node Law. They are employed here to reduce the number of variables in order to simply the problem in finding leakage inductances. Two techniques are in fact independent of each other, therefore it doesn't matter which to operate first. As we can see from our example, $W_{1} \& W_{3}$ are connected in parallel and $W_{2} \& W_{4}$ are connected in series, reduction of variables in equation (2) is certainly possible.

Let's start with the basic Series Reduction Technique from which we add the two voltages of the two winding elements in series and set their currents common

$$
\begin{aligned}
& v_{C}=v_{2}+v_{4} \\
& { }^{i_{C}}=i_{2}=i_{4}
\end{aligned}
$$

In our example, $W_{2} \& W_{4}$ are connected series which is equal to $W C$. Therefore two equations can describe the series reduction operation

$$
\begin{aligned}
& \left(\begin{array}{l}
v_{1} \\
v_{C} \\
v_{3} \\
v_{5}
\end{array}\right)=\left(\begin{array}{lllll}
1 & 0 & 0 & 0 & 0 \\
0 & 1 & 0 & 1 & 0 \\
0 & 0 & 1 & 0 & 0 \\
0 & 0 & 0 & 0 & 1
\end{array}\right) \cdot\left(\begin{array}{l}
v_{1} \\
v_{2} \\
v_{3} \\
v_{4} \\
v_{5}
\end{array}\right) \\
& \text { Matrix } A \\
& \left(\begin{array}{l}
i_{1} \\
i_{2} \\
i_{3} \\
i_{4} \\
i_{5}
\end{array}\right)=\left(\begin{array}{llll}
1 & 0 & 0 & 0 \\
0 & 1 & 0 & 0 \\
0 & 0 & 1 & 0 \\
0 & 1 & 0 & 0 \\
0 & 0 & 0 & 1
\end{array}\right) \cdot\left(\begin{array}{c}
i_{1} \\
i_{C} \\
i_{3} \\
i_{5}
\end{array}\right)
\end{aligned}
$$

Matrices $\boldsymbol{A}$ and $\boldsymbol{B}$ are trivial to find. By taking equation (5) and (6), equation (2) is now reduced to

$$
\begin{aligned}
\left(\begin{array}{l}
v_{1} \\
v_{C} \\
v_{3} \\
v_{5}
\end{array}\right)=s \cdot\left(\begin{array}{lllll}
1 & 0 & 0 & 0 & 0 \\
0 & 1 & 0 & 1 & 0 \\
0 & 0 & 1 & 0 & 0 \\
0 & 0 & 0 & 0 & 1
\end{array}\right) \cdot\left(\begin{array}{lllll}
L_{11} & M_{12} & M_{13} & M_{14} & M_{15} \\
M_{21} & L_{22} & M_{23} & M_{24} & M_{25} \\
M_{31} & M_{32} & L_{33} & M_{34} & M_{35} \\
M_{41} & M_{42} & M_{43} & L_{44} & M_{45} \\
M_{51} & M_{52} & M_{53} & M_{54} & L_{55}
\end{array}\right) \cdot\left(\begin{array}{llll}
1 & 0 & 0 & 0 \\
0 & 1 & 0 & 0 \\
0 & 0 & 1 & 0 \\
0 & 1 & 0 & 0 \\
0 & 0 & 0 & 1
\end{array}\right) \cdot\left(\begin{array}{c}
i_{1} \\
i_{C} \\
i_{3} \\
i_{5}
\end{array}\right) \quad(7) \\
\text { Reduced to a 4x4 Square Matrix } \boldsymbol{F}
\end{aligned}
$$

Moreover, Matrix $\boldsymbol{B}$ is actually the Transpose of Matrix $\boldsymbol{A}$ (i.e. $\boldsymbol{B}=\boldsymbol{A}^{T}$ ), which simplify the operation to

$$
v=s \cdot A \cdot L \cdot A^{T} \cdot i
$$

or simply

$$
v=s \cdot F \cdot i
$$


where

$$
F=A \cdot L \cdot A^{T}
$$

Now we can see that the Parallel Reduction is similar to Series Reduction where we add the two currents of the two winding elements in parallel and set their voltages common. The two transformation matrices deduced here are also in Transpose form. Refer to our example again, we have $W_{l} \&$ $W_{3}$ in parallel, thus we have

$$
\begin{aligned}
& { }^{i} A=i_{1}+i_{3} \\
& v_{A}=v_{1}=v_{3}
\end{aligned}
$$

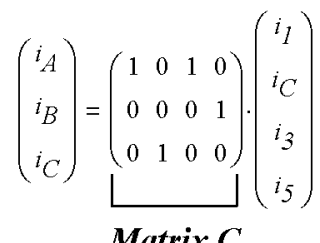

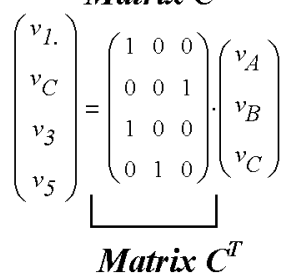

By taking the inverse of Matrix $\boldsymbol{F}$ we can then use equations (13) and (14) to reduce equation (9) to

$$
i=\frac{1}{s} \cdot C \cdot F^{-1} \cdot C^{T} \cdot v
$$

or simply

$$
i=\frac{1}{s} \cdot G \cdot v
$$

where

$$
G=C \cdot F^{-1} \cdot C^{T}
$$

Thus Matrix $G$ completely describes the inductive relationship of the transformer. Self inductances of $W A, W B$ and $W C$ can be easily deduced from Matrix $\boldsymbol{G}$. Let Matrix $\boldsymbol{H}$ equals the inverse of Matrix $G$ and rearranges equation (16), now elements $H_{11} H_{22}$ and $H_{33}$ are in fact the self inductances of $W A, W B$ and $W C$ respectively. Other elements in Matrix $\boldsymbol{H}$ correspond to the mutual inductances of the three windings

$$
\left(\begin{array}{c}
v_{A} \\
v_{B} \\
v_{C}
\end{array}\right)=s \cdot\left(\begin{array}{lll}
H_{11} & H_{12} & H_{13} \\
H_{21} & H_{22} & H_{23} \\
H_{31} & H_{32} & H_{33}
\end{array}\right) \cdot\left(\begin{array}{c}
i_{A} \\
i_{B} \\
i_{C}
\end{array}\right)
$$

Leakage inductance is determined by setting equivalent voltages for windings $W A, W B$, and $W C$. For example, to find the leakage inductance of winding $W A$, we apply the traditional short circuit method, for simplicity we set the voltage of $W B$ and $W C v_{B}=v_{c}=0$ and $v_{A}=1$

Substitute back to equation (16) to give

$$
\left(\begin{array}{c}
i_{A} \\
i_{B} \\
i_{C}
\end{array}\right)=\frac{1}{s} \cdot\left(\begin{array}{lll}
G_{11} & G_{12} & G_{13} \\
G_{21} & G_{22} & G_{23} \\
G_{31} & G_{32} & G_{33}
\end{array}\right) \cdot\left(\begin{array}{l}
1 \\
0 \\
0
\end{array}\right)
$$

From equation (19) the element $1 / G_{11}$ is essentially the leakage inductance of Winding $W A$, similarly $1 / G_{22}$ and $l / G_{33}$ are the leakage inductances of Winding $W B$ and $W C$ respectively. It is equally well to start with parallel operation first to reduce the variables involved, same result is yielded. Furthermore, we can also deduce for example the leakage of winding $W A$ with only WB in short circuit, for this case we simply truncate information associate with $W C$ from equation (18) and get

$$
\left(\begin{array}{c}
v_{A} \\
v_{B}
\end{array}\right)=s \cdot\left(\begin{array}{ll}
H_{11} & H_{12} \\
H_{21} & H_{22}
\end{array}\right) \cdot\left(\begin{array}{l}
i_{A} \\
i_{B}
\end{array}\right)
$$

By setting $v_{A}=1$ and $v_{B}=0$ and taking inverse of the $2 \times 2$ matrix formed by $H_{11}, H_{12}, H_{21}$ and $H_{22}$ (denote the inverse of this as Matrix $J$ ), then the element $1 / J_{11}$ is essentially the leakage inductance of Winding $W A$ with only $W B$ in short circuit. Thus the element $1 / J_{22}$ is the leakage inductance of winding $W B$ with only $W A$ in short circuit. Similarly other combination (i.e. $W A \& W C, W B \& W C$ ) can be implemented in this way.

\section{EXPERIMENTAL VERIFICATION}

For easy understandings we will compare the calculated values of leakage inductance from Matrix $G$ of our example shown in Fig.1 with the measured leakage inductance values performed with short-circuit test using an HP 4192A impedance analyzer. The transformer is constructed with a NEC-Tokin FEER28L Horizontal Bobbin with a diameter of $12 \mathrm{~mm}$ and air core. Standard AWG 25 single coated wire with a diameter of $0.4928 \mathrm{~mm}$ is selected for all tests. We set $W_{1}=W_{2}=W_{3}=40$ Turns, $W_{4}=15$ Turns and $W_{5}=25$ Turns, distributed evenly around the winding area. Self inductance values and mutual inductance values of all combination of two winding elements are deduced from Chapter 15 and Chapter 16 of [3] respectively. Four layers of 0.2 mil is used to separated each layer to ensure a more rigid layer construction. Three sets of results are provided for verification. Table 1 shows the calculated and measured self inductance values of windings $W A, W B$ and $W C$, where calculated values are deduced from Matrix $\boldsymbol{H}$ as described above. Second set values are deduced from Matrix $\boldsymbol{J}$ with only one winding in short circuit. Lastly the leakage inductances of $W \mathrm{~A}, W B$ and $W C$ are calculated from Matrix $\boldsymbol{G}$ with all other windings shorted. High frequency factor is 
not taken into account when calculating Matrix $\boldsymbol{L}$, therefore real measurements $a, 20 \mathrm{kHz}$ is chosen for verification purpose where the impedance analyzer can give us reasonably accurate results at this region without much deviation to DC inductance due to high frequency effects. Results demonstrate a good match with a percentage error of less than $15 \%$ with most results lies within $8 \%$.

\begin{tabular}{|c|c|c|c|c|}
\hline $\begin{array}{c}\text { Self } \\
\text { Inductance } \\
\text { from } \\
\text { Combined } \\
\text { Winding } \\
\text { Elements } \\
\end{array}$ & $\begin{array}{c}\text { Calculated } \\
\text { Value } \\
\text { deduced } \\
\text { from Matrix } \\
H \text { (Henrys) }\end{array}$ & $\begin{array}{c}\text { Measured } \\
\text { Value @ } \\
20 k H z \\
\text { (Henrys) }\end{array}$ & $\begin{array}{c}\text { Error } \\
\text { (Henrys) }\end{array}$ & $\begin{array}{c}\% \\
\text { Error }\end{array}$ \\
\hline WA & $9.21 \mathrm{E}-06$ & $9.41 \mathrm{E}-06$ & $-2.04 \mathrm{E}-07$ & -2.16 \\
\hline$W B$ & 6.70E-06 & 7.25E-06 & $-5.50 \mathrm{E}-07$ & -7.59 \\
\hline \multirow[t]{2}{*}{$W C$} & $2.04 \mathrm{E}-05$ & $2.08 \mathrm{E}-05$ & $-3.46 \mathrm{E}-07$ & -1.66 \\
\hline & $\begin{array}{l}\text { Deduced } \\
\text { from Matrix } \\
\mathbf{J}\end{array}$ & & & \\
\hline $\begin{array}{l}\text { WA (Short } \\
\text { WB ONLY) }\end{array}$ & $4.54 \mathrm{E}-06$ & $4.40 \mathrm{E}-06$ & 1.34E-07 & 3.03 \\
\hline $\begin{array}{l}\text { WA (Short } \\
\text { WC ONLY) }\end{array}$ & 1.75E-06 & 1.70E-06 & $5.09 \mathrm{E}-08$ & 2.99 \\
\hline $\begin{array}{l}\text { WB (Short } \\
\text { WA ONLY) }\end{array}$ & 3.30E-06 & $3.27 \mathrm{E}-06$ & 2.73E-08 & 0.83 \\
\hline $\begin{array}{l}\text { WB (Short } \\
\text { WC ONLY) }\end{array}$ & $4.78 \mathrm{E}-06$ & $4.88 \mathrm{E}-06$ & $-1.05 \mathrm{E}-07$ & -2.15 \\
\hline $\begin{array}{l}\text { WC (Short } \\
\text { WA ONLY) }\end{array}$ & 3.89E-06 & 3.62E-06 & $2.72 \mathrm{E}-07$ & 7.51 \\
\hline \multirow[t]{2}{*}{$\begin{array}{l}\text { WC (Short } \\
\text { WB ONLY) }\end{array}$} & $1.46 \mathrm{E}-05$ & $1.42 \mathrm{E}-05$ & $3.55 \mathrm{E}-07$ & 2.50 \\
\hline & $\begin{array}{c}\text { Deduced } \\
\text { from Matrix } \\
\mathbf{G} \\
\end{array}$ & & & \\
\hline $\begin{array}{l}W A \text { (Short } \\
W B \& W C \text { ) }\end{array}$ & $1.07 \mathrm{E}-06$ & $1.02 \mathrm{E}-06$ & $4.18 \mathrm{E}-08$ & 4.08 \\
\hline $\begin{array}{l}\text { WB (Short } \\
W A \& W C \text { ) }\end{array}$ & $2.91 \mathrm{E}-06$ & 3.34E-06 & $-4.30 \mathrm{E}-07$ & -12.8 \\
\hline $\begin{array}{l}W C \text { (Short } \\
W A \& W B \text { ) }\end{array}$ & $3.43 \mathrm{E}-06$ & $3.47 \mathrm{E}-06$ & $-4.51 E-08$ & -1.30 \\
\hline
\end{tabular}

Table 1

\section{CONCLUSION}

The method presented above provides a platform in calculating complex constructed transformer structure based on a two-winding mutual inductance model. It greatly simplifies the calculation as we do not need to handle complex relationships of all transformer windings in one go, only the mutual inductances between pairs of winding elements are necessary. One can imagine that for any transformer that is winded concentrically, this method can be applied equally well and make the leakage inductance calculations trivial. Its robustness with a systematic way of matrix operations provides a new dimension in viewing the problem. In essence, the matrix operation reduces many difficult calculations into one part. That is the accurate calculations of mutual inductances for any two winding elements and self inductances of a winding element at a particular frequency where interested. Future works include the development of the model of modification factors at high frequency case with skin effect and proximity effect taken into account. The approach has been implemented on
PowerESIM [5][6], a web base server type power converter design program, for reader to test.

\section{ACKNOWLEDGMENT}

The authors would like to thank Mr. Joe C. P. Liu and Dr. Franki N. K. Poon for their valuable comments and suggestions.

\section{REFERENCES}

[1] A. Schellmanns, K. Berrouche, J. Keradec, "Multiwinding Transformers: A Successive Refinement Method to Characterize a General Equivalent Circuit", IEEE Transaction on Instrumentation and Measurement, Vol. 47, No. 5, Oct 1998.

[2] L. Heinemann, J. Helfrich "Modelling and Accurate Determination of Winding Losses of High Frequency Transformers in Various Power Electronics Applications", Applied Power Electronics Conference and Exposition, 2000. APEC 2000. Fifteenth Annual IEEE Volume 2, 6-10 Feb. 2000 Page(s):647 - 653 vol.2

[3] F. W. Grover, "Inductance Calculations, working formulas and Tables", Dover Publications, Inc., New York, N. Y., Special edition prepared for the Instrument Society of America, 1973.

[4] L. O. Chua, C. A. Desoer, E. S. Kuh, "Linear and Nonlinear Circuits", International Edition, McGRAW-HILL Series, 1987.

[5] www.poweresim.com

[6] N. K. Poon, "Component-Based System for Designing Power Supply of no Expert Knowled ge Required", Accepted for presentation in PESC06, June 2006. 